Cartas científicas

Rev Urug Cardiol 2021; 36: e703

doi: $10.29277 /$ cardio.36.3.15

\title{
Miopericarditis aguda versus síndrome coronario agudo: reporte de un caso
}

\section{Resumen}

La miopericarditis aguda es una pericarditis aguda con compromiso miocárdico menor. En las primeras horas de evolución el cuadro clínico puede confundirse con un síndrome coronario agudo, en especial cuando el electrocardiogr cvama presenta elevación del segmento ST y marcadores de necrosis miocárdica elevados. La resonancia magnética cardíaca tiene un papel importante para diferenciar las dos enfermedades. Presentamos el caso de un hombre de 36 años de edad ingresado por dolor de pecho, elevación del segmento ST y de marcadores de necrosis miocárdica. Se realiza angiografía coronaria, que no evidencia lesiones angiográficamente significativas, resonancia magnética cardíaca con contraste de gadolinio, en la que se observa leve derrame pericárdico, presencia de edema y realce tardío con patrón parcheado subepicárdico e intramiocárdico en pared lateral. Los hallazgos del estudio confirman el diagnóstico de miopericarditis.

Palabras clave: MIOPERICARDITIS

SÍNDROME CORONARIO AGUDO RESONANCIA MAGNÉTICA CARDÍACA

\section{Acute myopericarditis versus acute coronary syndrome: case report}

\section{Summary}

Acute myopericarditis is an acute pericarditis with minor myocardial compromise. During the first hours from onset the clinical presentation can be confused with an acute coronary syndrome, especially when the electrocardiogram presents with ST segment elevation and increased markers of myocardial necrosis. Cardiac magnetic resonance imaging plays an important role to differentiate between these two diseases. We present the case of a 36-year-old male who was admitted with chest pain, ST segment elevation and increased markers of myocardial necrosis. Coronary angiography is performed reporting no significant angiographic findings. Cardiac magnetic resonance imaging with contrast medium (gadolinium) is performed, reporting mild pericardial effusion, presence of edema, and delayed uptake with a subepicardial and intramyocardial patchy pattern on the lateral wall. These test findings confirm the diagnosis of myopericarditis.

Key words: $\quad$ MYOPERICARDITIS

\section{ACUTE CORONARY SYNDROME}

CARDIAC MAGNETIC RESONANCE

\section{Miopericardite aguda versus síndrome coronariana aguda: relato de um caso}

\section{Resumo}

A miopericardite aguda é uma pericardite aguda com pequena deterioração miocárdica. Nas primeiras horas de evolução do quadro clínico podem ser confundidas com síndrome coronariana aguda, principalmente quando o eletrocardiograma mostra supradesnivelamento do segmento ST e marcadores elevados de necrose miocárdica. A ressonância magnética cardíaca desempenha um papel importante em distinguir as duas doenças. Apresentamos o caso de um homem de 36 anos admitido por dor torácica, supradesnivelamento do segmento ST e elevação dos marcadores de necrose miocárdica, foi realizada cineangiocoronariografia, onde não foram evidenciadas lesões angiograficamente significativas. Foi realizada a ressonância magnética cardíaca com contraste de gadolínio e foram observados derrame pericárdico leve, edema e realce tardio com um padrão irregular subepicárdico e intramiocárdico na face lateral. Os resultados do estudo confirmam o diagnóstico de miopericardite.

Palavras chave: MIOPERICARDITE SÍNDROME CORONARIANA AGUDA RESSONÂNCIA MAGNÉTICA CARDÍACA

Sr. Editor:

\section{Introducción}

La miopericarditis aguda es el cuadro clínico de pericarditis aguda con elevación de marcadores de necrosis miocárdica (troponina I o T o CK-MB) sin deterioro focal o difuso de la función ventricular izquierda evaluado por ecocardiografía o resonancia magnética cardíaca $(\mathrm{RMC})^{(1)}$.

La incidencia exacta y la prevalencia de miopericarditis no se han establecido. La pericarditis aguda es el diagnóstico de ingreso en el 0,1\% de los ingresos hospitalarios. Se estima que la incidencia 


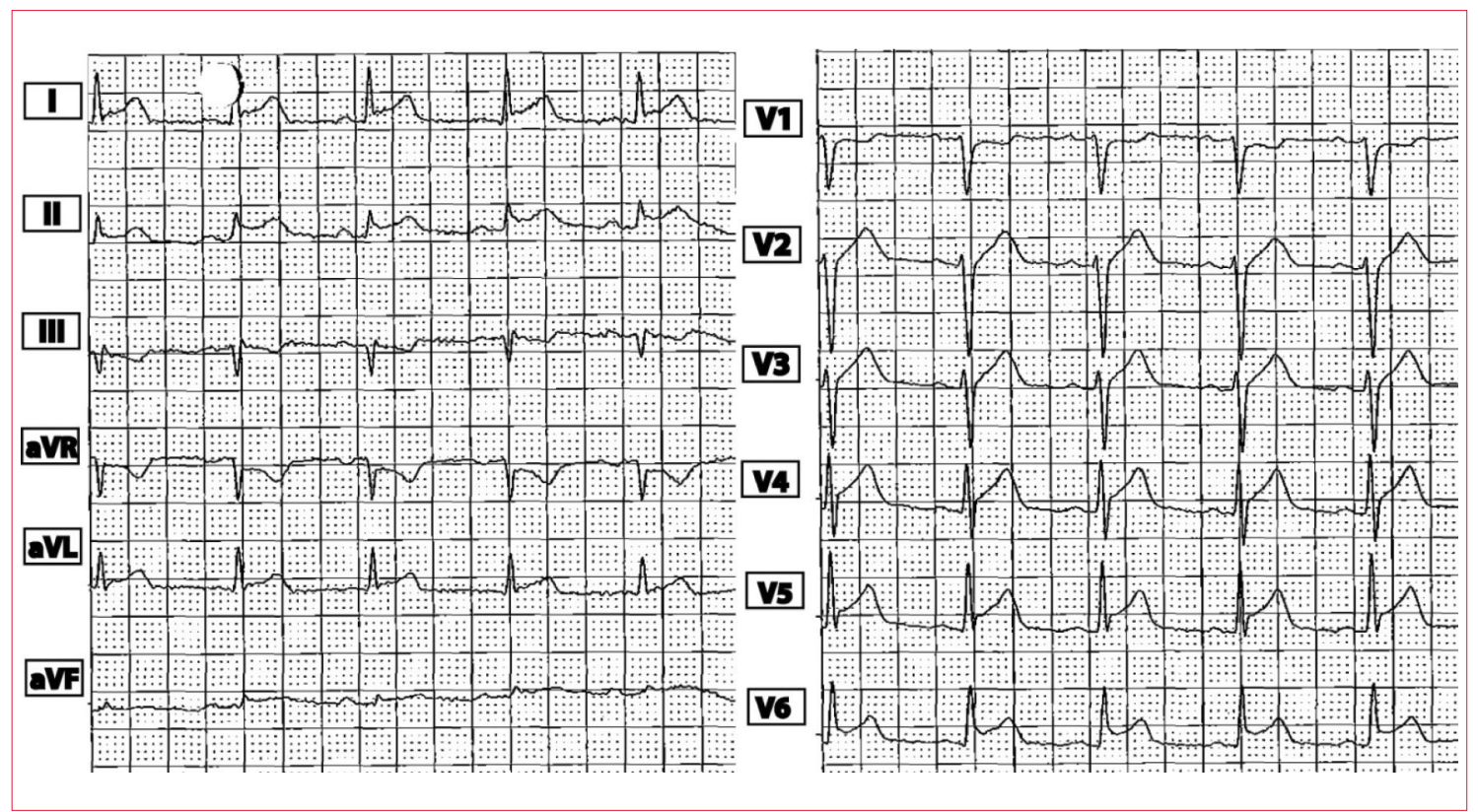

Figura 1. ECG de emergencia: ritmo sinusal, intervalos conservados, elevación cóncava del segmento ST en paredes inferior y lateral.

de miocarditis es de alrededor de 1 a 10 casos por cada 100.000 personas/año(2).

La miocarditis y la pericarditis comparten agentes etiológicos similares, las infecciones virales son la causa más frecuente en países desarrollados. Entre las causas bacterianas, la tuberculosis es una causa importante en todo el mundo, debido al alto número de casos en países en desarrollo, en especial en el contexto de la coinfección por el virus de inmunodeficiencia humana (VIH) ${ }^{(3)}$.

En los casos sospechosos de miopericarditis, se recomienda realizar angiografía coronaria (según la presentación clínica y la evaluación de factores de riesgo) para descartar un síndrome coronario agudo. La RMC está recomendada para confirmar la afección miocárdica y descartar la necrosis miocárdica isquémica en ausencia de enfermedad coronaria significativa ${ }^{(3)}$.

\section{Caso clínico}

Paciente masculino de 36 años de edad, sin antecedentes patológicos de importancia. El cuadro inicia 9 días previos, con enfermedad tipo viral (rinorrea, tos, fiebre, malestar general), con tratamiento ambulatorio. Acude a sala de emergencia con cuadro de 30 minutos de evolución, caracterizado por dolor precordial tipo opresivo de elevada intensidad, irradiado a miembro superior izquierdo que aumenta a la inspiración profunda, náuseas, vómito, sudoración profusa y disnea. En la auscultación cardíaca, se constata ritmo regular, sin soplos ni frote pericárdico y el resto del examen físico sin particularidades. Se realiza electrocardiograma (ECG) que evidencia elevación del segmento ST en paredes inferior y lateral (figura 1).

El laboratorio revela movimiento enzimático (troponina I 88,7 ng/ml, CK-MB 89,9 U/L, CPK 1358 U/L), velocidad de sedimentación globular (VSG) 30 $\mathrm{mm} / \mathrm{h}$ y proteína C reactiva (PCR) $12 \mathrm{mg} / \mathrm{L}$. Con la sospecha de un síndrome coronario agudo se realiza angiografía coronaria de emergencia. El estudio no evidencia lesiones angiográficamente significativas.

$\mathrm{El}$ ecocardiograma muestra un aumento discreto del atrio izquierdo, contractilidad miocárdica del ventrículo izquierdo con hipocinesia difusa discreta, ausencia de derrame pericárdico, fracción de eyección de $51 \%$.

Ocurre un descenso de las enzimas cardíacas a las 6 horas del ingreso, sin cambios en el electrocardiograma.

En la RMC con contraste de gadolinio se observa hipocinesia global discreta del ventrículo izquierdo, fracción de eyección de $54 \%$, leve derrame pericárdico, presencia de edema y realce tardío con patrón parcheado subepicárdico e intramiocárdico en pared lateral (figura 2).

Se llega al diagnóstico de miopericarditis aguda de probable etiología viral y se inicia tratamiento con ibuprofeno $600 \mathrm{mg}$ cada 8 horas, colchicina 0,5 mg cada 12 horas, con buena evolución clínica y laboratorial. Se decide alta hospitalaria a las 72 horas del ingreso. Se recomienda restricción de la actividad física por lo menos por 3 meses y seguimiento 


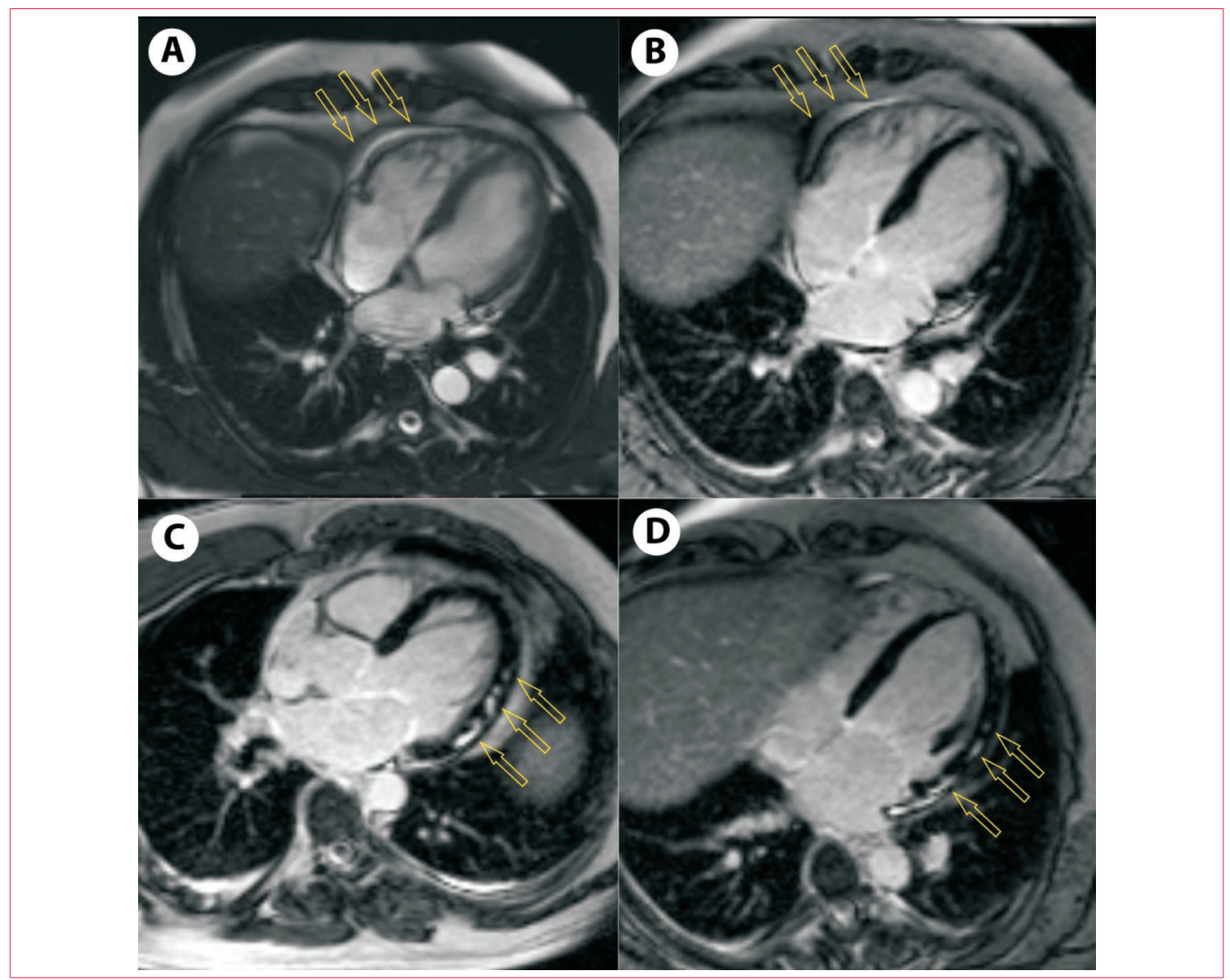

Figura 2. Resonancia magnética cardíaca con gadolinio. A) y B) Derrame pericárdico (flechas). C) y D) Realce tardío con patrón parcheado subepicárdico o intramiocárdico en pared lateral (flechas).

por consulta externa de cardiología a las 4 y 12 semanas.

En el seguimiento por consulta externa a las 4 semanas se realiza electrocardiograma que evidencia ondas T negativas en paredes inferior y lateral (figura 3). A las 12 semanas un nuevo electrocardiograma evidencia positivización de las ondas $\mathrm{T}$ (figura 4).

\section{Discusión}

La presentación clínica refleja el grado de afectación del pericardio, del miocardio y la agudeza de la enfermedad. Los pacientes pueden presentar un pródromo viral de 1 a 2 semanas antes de la presentación de los primeros síntomas. Estos síntomas incluyen disnea, palpitaciones, fiebre y dolor de pecho continuo que aumenta con la inspiración y alivia al sentarse hacia adelante, puede irradiarse al hombro izquierdo o mandíbula, difícil de diferenciar del dolor de isquemia miocárdica. Los hallazgos del examen físico pueden ser variables, pero los más comunes pueden incluir fiebre, fricción pericárdica y características de insuficiencia cardíaca $^{(4)}$.

Los cambios electrocardiográficos son comunes. Un patrón típico de evolución del ECG incluye elevación difusa cóncava del segmento ST, depresión del PR, seguida de normalización del segmento ST y PR, y luego inversión difusa de las ondas T. Provoca elevación de marcadores inflamatorios (VSG, PCR) y marcadores de necrosis miocárdica. La biopsia miocárdica y las serologías virales no son útiles en el proceso diagnóstico y terapéutico de las miopericarditis no complicadas ${ }^{(4)}$.

En los casos sospechosos de miopericarditis, se recomienda realizar angiografía coronaria (según la presentación clínica y la evaluación de factores de riesgo) para descartar un síndrome coronario agudo. La RMC está recomendada para confirmar afección miocárdica y descartar la necrosis miocárdica isquémica en ausencia de enfermedad coronaria significativa. El derrame pericárdico y el realce tardío de gadolinio con patrón parcheado subepicárdico e intramiocárdico junto con edema miocárdico en diferentes territorios vasculares es indicativo de miopericarditis ${ }^{(3,6)}$. 


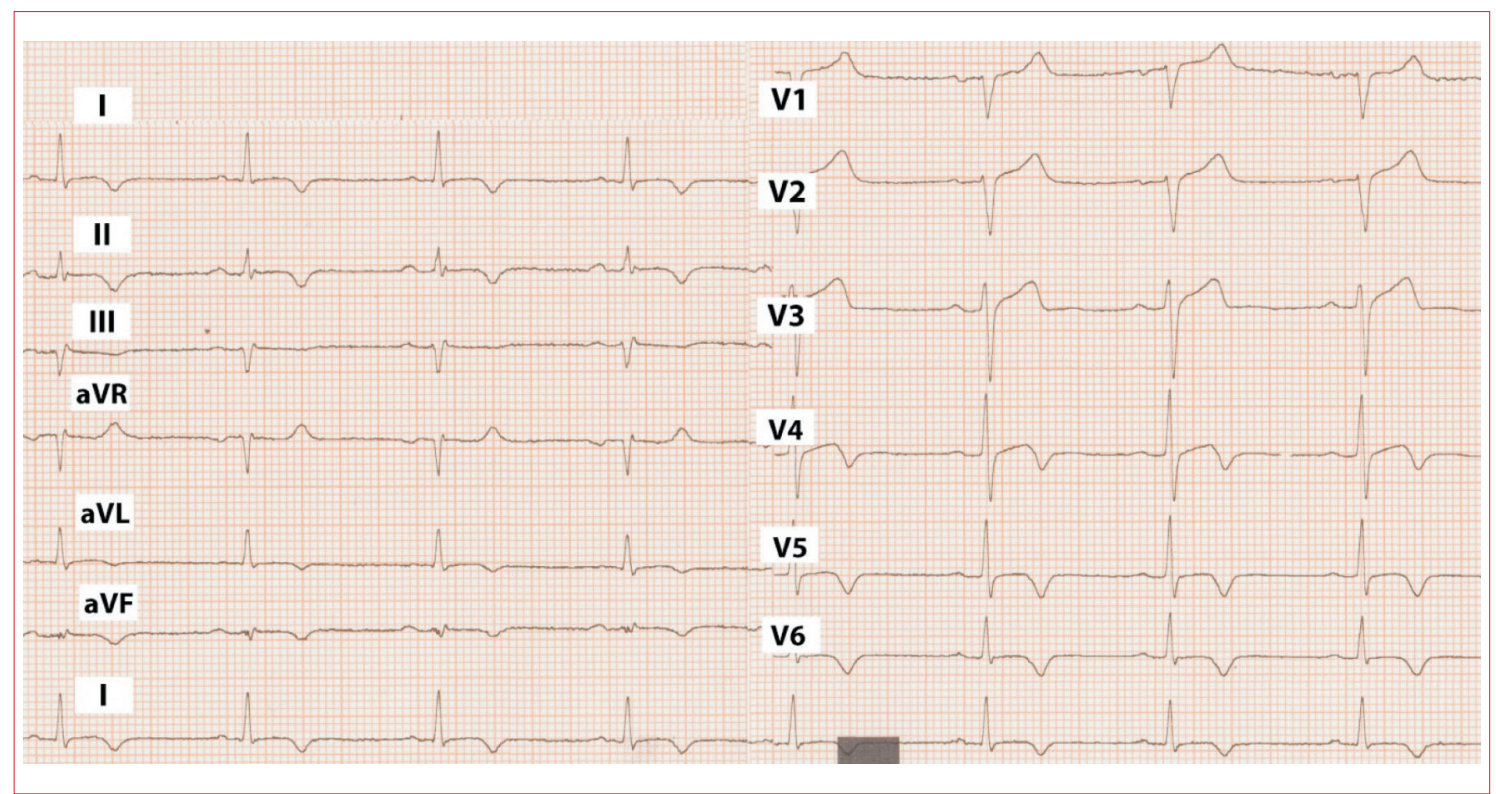

Figura 3. ECG control 4 semanas: bradicardia sinusal, intervalos conservados, ondas T negativas en paredes inferior y lateral.

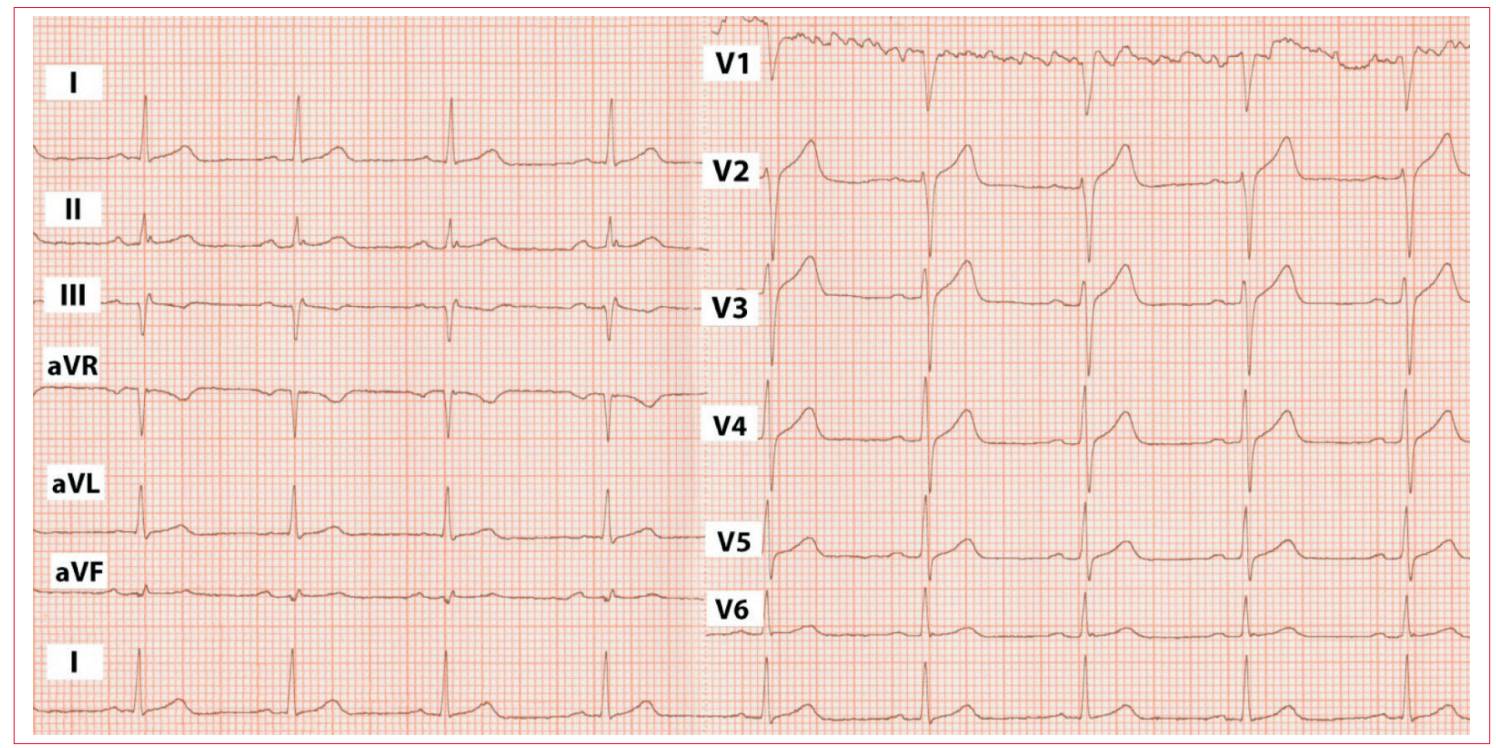

Figura 4. ECG control 12 semanas: ritmo sinusal, intervalos conservados, sin alteraciones del segmento ST y onda T.

El manejo es similar al recomendado para la pericarditis: se suele prescribir aspirina (1.500$3.000 \mathrm{mg} /$ día) o AINE (ibuprofeno 1.200-2.400 $\mathrm{mg} /$ día o indometacina 75-150 mg/día) para controlar el dolor torácico, mientras que los corticoides se prescriben como segunda opción en casos de contraindicación, intolerancia o fracaso de la aspirina o AINE. No hay datos suficientes para recomendar el uso de colchicina, que es un tratamiento adyuvante bien establecido para la pericarditis aguda y recurrente. Se recomienda reposo y evitar cualquier ejercicio físico más allá de las actividades sedentarias normales a los no deportistas y los deportistas con miopericarditis, durante un período de 6 meses $^{(3,4)}$.

La miopericarditis tiene buen pronóstico general. Las complicaciones pericárdicas específicas (recurrencia, taponamientos cardíacos, pericarditis constrictiva) son raras. La elevación de troponina no predice un resultado adverso en la mayoría de los casos. Varias series observacionales han demostrado la ausencia de evolución hacia insuficiencia cardíaca o muerte ${ }^{(7)}$. 


\section{Conclusión}

El relato del caso enfatiza la importancia de la $\mathrm{RMC}$ en el diagnóstico diferencial entre miopericarditis aguda y un síndrome coronario agudo. Consideramos que es un método no invasivo de gran relevancia para establecer el diagnóstico de miopericarditis aguda, en especial cuando exámenes iniciales como ecocardiograma y angiografía coronaria no son capaces de constatar alteraciones que justifiquen el cuadro clínico.

Osman Guevara Manzano ${ }^{1}$, Fernando Leite Vincenti ${ }^{2}$ 1. Servicio de Cardiología, Hospital Universitario Hernández Vera. Santa Cruz, Bolivia. 2. Servicio de Cardiología, Clínica Foianini. Santa Cruz, Bolivia. Correspondencia: Dr. Osman Guevara Manzano. Correo electrónico: osmaneduardo77@gmail.com

Osman Guevara Manzano, https://orcid.org/0000-0003-1003-4223

Fernando Leite Vincenti, https://orcid.org/0000-0002-2061-9308

Este artículo fue aceptado para su publicación por el Editor, Dr. Jorge Estigarribia

\section{Bibliografía}

1. Casabé JH, Klin P, Oberti P, Trivi M, Vázquez Blanco M. Consenso Enfermedades del Pericardio. Rev Argent Cardiol. 2017 Dic [consulta:2 Set 2021];85 Supl 7:(aprox.55p.). Disponible en: https://www. sac.org.ar/wp-content/uploads/2018/ 08/consensode-pericardio-2018.pdf.

2. Manda YR, Baradhi KM. Myopericarditis. StatPearls Treasure Island (FL): StatPearls; 2021 [consulta: 1 Jul 2021]. Disponible en: https://www.ncbi. nlm.nih.gov/books/NBK534776/.

3. Adler Y, Charron P, Imazio M, Badano L, Barón-Esquivias G, Bogaert J, et al. 2015 ESC Guidelines for the diagnosis and management of pericardial diseases: The Task Force for the diagnosis and management of pericardial diseases of the European Society of Cardiology (ESC) endorsed by: The European Association for Cardio-Thoracic Surgery
(EACTS). Eur Heart J. 2015;36(42):2921-64. doi: 10. 1093/eurheartj/ehv318.

4. Farzad A, Schussler JM. Acute myopericardial syndromes. Cardiol Clin. 2018;36(1):103-14. doi: 10.1016/j.ccl.2017.09.004.

5. Imazio M, Cooper LT. Management of myopericarditis. Expert Rev Cardiovasc Ther. 2013; 11(2):193-201. doi: 10.1586/erc.12.184

6. Valbuena-López S, Hinojar R, Puntmann VO. Cardiovascular magnetic resonance in cardiology practice: a concise guide to image acquisition and clinical interpretation. Rev Esp Cardiol (Engl Ed). 2016; 69(2):202-10. doi:10.1016/j.rec.2015.11.011.

7. Imazio M, Brucato A, Spodick DH, Adler Y. Prognosis of myopericarditis as determined from previously published reports. J Cardiovasc Med (Hagerstown). 2014;15(12):835-9. doi:10.2459/JCM.0000000000000082 . 